\title{
PROVA DE CONCEITO SOBRE IMPACTOS EPISTÊMICOS E ONTOLÓGICOS NA INTEROPERABILIDADE SEMÂNTICA DE TERMINOLOGIAS CLÍNICAS
}

\author{
Jeanne Louize Emygdio e Maurício Barcellos Almeida \\ Universidade Federal de Minas Gerais, Brasil
}

\begin{abstract}
RESUMO
Apresenta-se uma pesquisa em andamento cujo propósito é o de demonstrar por meio de uma prova de conceito, que abordagens ontológicas, para fins de interoperabilidade semântica, podem apresentar vantagens sobre aquelas que se valem de abordagens puramente linguísticas. A metodologia envolve uso de métodos léxicos e estruturais para combinar, mapear, alinhar e integrar terminologias clínicas, considerando amostras de vocabulários, precedidos ou não de escrutínio ontológico. Nesse momento da pesquisa, prevê-se uma análise qualitativa dos dados através da mensuração do grau de eficiência de cada abordagem - linguística ou ontológica - para prover integração entre as terminologias clínicas. Espera-se obter contribuições teóricas e práticas que permitam a descoberta e distinção dos aspectos ontológicos e epistêmicos inerentes às terminologias clínicas, e elucidem os desafios epistêmicos à interoperabilidade semântica, além de destacar a relevância da abordagem ontológica para mitigar problemas de interoperabilidade. Espera-se obter uma ontologia computacional de integração entre o SNOMED CT e a CID no âmbito da obstetrícia, documentada, a ser distribuída publicamente em formato aberto.
\end{abstract}

\section{PALAVRAS-CHAVE}

Interoperabilidade Semântica, Terminologias Clínicas, Representação do Conhecimento, Ontologias Formais

\section{INTRODUÇÃO}

Ecossistemas de saúde são domínios complexos onde elementos humanos, técnicos, processuais e regulatórios interagem, participando de práticas de busca por interoperabilidade sob diversas formas, nem sempre conhecidas ou bem compreendidas (ABNT, 2016; SCHULZ et al, 2017). Como resultado desta interação, uma grande quantidade de dados heterogêneos vem sendo criada e armazenada em silos de dados (SMITH, 2008) já há décadas, principalmente no domínio da Medicina. A articulação destes recursos durante a prestação do cuidado à saúde pode possibilitar um acesso consensual a dados, permitindo a construção de Prontuários Eletrônicos de Pacientes (PEPs) um mínimo integrados.

Com o propósito de uniformizar a comunicação no contexto médico, diversas organizações concorrem para o provimento de padrões, com destaque para a International Organization for Standardization (ISO) ${ }^{1}$ e a Health Level Seven International (HL7) ${ }^{2}$, para citar algumas. Assim, diversos padrões acabam por concorrer para alcançar, às vezes os mesmos fins, como por exemplo: SNOMED CT, CID, HL7, openEHR ${ }^{3}$, entre outros, resultando em sobreposições e redundâncias recorrentes, as quais, acrescidas da natureza distinta de cada padrão, comprometem a possibilidade de interoperabilidade no contexto das instituições de saúde (Schulz, Stegwee e Chronaki, 2019).

A presente pesquisa aborda questões que dificultam a interoperabilidade semântica no contexto médico. Para tal, adotam-se as seguintes premissas:

i) a expressão "terminologias clínicas" (Schulz et al, 2017) é adotada como referência aos padrões terminológicos, a despeito da diversidade de suas nomenclaturas;

\footnotetext{
Maiores informações em: https://www.iso.org/home.html. Acesso em: 16/09/2019.

Maiores informações em: https://www.hl7.org/. Acesso em: 16/09/2019.

3 Maiores informações em: https://www.openehr.org/what_is_openehr. Acesso em 06/07/2019.
} 
ii) na Ciência da Computação, o volume expressivo de pesquisas neste entorno (Ochieng e Kyanda, 2018) direciona-se ao provimento de técnicas e ferramentas de alinhamento semântico de terminologias médicas (Euzenat e Shvaiko, 2007); na Ciência da Informação a ênfase recai na descoberta e distinção dos aspectos ontológicos e epistêmicos que permeiam as classificações, os desafios que a epistemologia impõe ao alcance de interoperabilidade semântica (Bodenreider, Smith e Burgun, 2004) e a forma como os fundamentos ontológicos podem ser empregados para mitigar tais problemas (Jansen, 2008; Smith, 2003);

iii) duas abordagens são de interesse para os propósitos desse artigo com respeito a integração de terminologias clínicas:

a) a utilizada pelo Sistema de Linguagem Médica Unificado (UMLS), baseada na identificação de relações de sinonímia entre termos;

b) a de padronização prospectiva, desenvolvida pelo OBO Foundry (Smith et al., 2007) através do BioPortal aderente a princípios compartilhados para construção e distribuição de ontologias.

$\mathrm{Na}$ literatura científica encontram-se estudos relacionados à adoção do escrutínio ontológico em terminologias clínicas e voltados à melhoria na representação do conhecimento a partir da:

i) correção de anomalias estruturais, definição de conceitos, relações e meta-classes (Schulz; Suntisrivaraporn e Baader, 2007; Bodenreider; Smith e Burgun, 2004);

ii) melhoria na eficiência para descoberta de combinações terminológicas e economia de recursos de processamento em função do alinhamento a partir de ontologias de alto nível (Zhang e Bodenreider, 2005);

iii) melhoria na identificação de fronteiras de conhecimento compartilhado entre terminologias clínicas visando sua harmonização para fins de mapeamento e evolução, tendo por base a definição de axiomas em linguagem OWL (Rodrigues et al, 2015).

iv) Por outro lado, maior efetividade para detecção de erros estruturais e de classificação em terminologias clínicas podem ser obtidos a partir do uso de técnicas híbridas de métodos léxicos e estruturais (Bodenreider, 2018).

Apresenta-se então a pesquisa em andamento cujo propósito é demonstrar por meio de uma prova de conceito que abordagens ontológicas podem ser mais efetivas para fins de interoperabilidade semântica do que as que adotam práticas puramente linguísticas. Nesse momento, em que a pesquisa ainda se encontra em uma fase qualitativa, não se definiu exatamente em que aspectos tal efetividade vai se manifestar.

A metodologia envolve uso de métodos léxicos e estruturais para combinar, mapear, alinhar e integrar terminologias clínicas, precedidos ou não do escrutínio ontológico. Prevê-se uma análise qualitativa dos dados, através da mensuração do grau de eficiência de cada abordagem para prover integração entre as terminologias clínicas.

Espera-se obter contribuições teóricas e práticas que permitam a descoberta e distinção dos aspectos ontológicos e epistêmicos inerentes às terminologias clínicas, destaquem os desafios epistêmicos à interoperabilidade semântica e demonstrem a relevância da abordagem ontológica para mitigar problemas de interoperabilidade. Espera-se obter uma ontologia computacional de integração entre o SNOMED CT e a CID no âmbito da obstetrícia, documentada, a ser distribuída publicamente em formato aberto.

\section{PESQUISA EM ANDAMENTO}

A presente pesquisa busca responder a seguinte questão: De que forma ontologias podem contribuir para resolver questões que impedem a interoperabilidade semântica?

Seu objetivo geral é o de testar a integração de terminologias clínicas a partir de uma abordagem ontológica e a partir de uma abordagem linguística, verificando qual é a mais efetiva para fins de interoperabilidade semântica. Como objetivos específicos busca-se:

i) identificar e distinguir os aspectos ontológicos e epistêmicos presentes nas terminologias clínicas;

ii) identificar âncoras léxicas que estabeleçam fronteiras compartilhadas de conhecimento entre as terminologias clínicas;

iii) identificar relações semânticas que possibilitem uma representação explícita do conhecimento que permeia as conexões entre as âncoras léxicas através da extração de relações hierárquicas; 
iv) identificar âncoras estruturais, através da comparação das relações semânticas previamente identificadas;

v) demonstrar o grau de efetividade alcançado por cada tipo de abordagem, tendo como base o número de combinações terminológicas encontradas aliado ao percentual de evidências positivas para interoperabilidade que estes resultados representam.

\subsection{Metodologia}

Nesta seção serão descritos aspectos metodológicos adotados para definição das amostras, as justificativas e o processo completo de execução da pesquisa.

\subsubsection{Definição das Amostras}

O universo da pesquisa compreende as terminologias clínicas. A unidade de análise compreende os conjuntos de termos relacionados à área obstétrica e neonatal. Tal domínio foi selecionado por conveniência, seja por conta de pesquisas anteriores, seja pelo acesso a um Depto. de Obstetrícia.

Foram identificados 32 terminologias clínicas e três repositórios de ontologias de interesse para a pesquisa, conforme apresentado na Tabela 1.

Tabela 1. Terminologias clínicas e repositórios de ontologias selecionados para a pesquisa

\begin{tabular}{clll}
\hline QTD. TIPO & QUAIS & SEMÂNTICA \\
\hline 05 & Taxonomias & ATC/DDD, CPT-4, DSM, HCPCS, ICD/CID, NANDA, & Linguagem natural \\
& & ISO9999 & Linguagem natural \\
06 & Tesauros & ICECI, ICF, ICHI, MedDRA, MeSH, NCIt & Mista \\
01 & Meta-tesauros & SNOMED-CT & Formal \\
03 & Frameworks & openEHR, HL7, IHE-PIX & Mista \\
04 & Padrões técnicos & TISS, DICOM, LOINC, ISBT 128 & Formal \\
13 & Ontologias & BWW, DOLCE, UFO, GFO, SOWA, BFO, OntONeo, GO, & \\
& & CL, FMA, IAO, OBI, OGMS & Formal \\
03 & Repositórios ontológicos & OBO Foundry, UMLS, Bioportal & \\
\hline
\end{tabular}

Selecionou-se a SNOMED CT, a CID e a OntoNEO, em função da perspectiva de contribuição social real ao país, a partir da:

i) possibilidade de replicação do experimento base (Zhang e Bodenreider, 2005);

ii) maturidade do repositório $O B O$ Foundry em construção de ontologias biomédicas;

iii) ortogonalidade da ontologia OntoNEO ao repositório mencionado e de sua tradução para o português, e,

iv) presença de especialistas para validação dos resultados.

Foram identificadas até o momento amostras estratificadas por classes e propriedades, conforme apresentado na Tabela 2.

Tabela 2. Amostras estratificadas por classes e propriedades relacionadas ao domínio obstétrico e neonatal

\begin{tabular}{l|c|c|c|c}
\hline \multirow{2}{*}{$\begin{array}{c}\text { TERMINOLOGIA } \\
\text { CLÍNICA }\end{array}$} & \multicolumn{2}{c|}{ GERAIS } & \multicolumn{2}{c}{ SELECIONADAS } \\
\cline { 2 - 5 } & Classes & Propr. & Classes & Propriedades \\
\hline SNOMED CT & 347,077 & 216 & \multicolumn{2}{c}{ Iniciando estudos } \\
\hline CID & 14.502 & 1 & $\begin{array}{c}\text { 3 Capítulos (XV, XVI, XVII); 222 Categorias (O00-O99; } \\
\text { P00-P96;Q00-Q99); 1.381 Sub-categorias. }\end{array}$ \\
\hline OntoNEO & 1.767 & 451 & 1.767 & 451 \\
\hline
\end{tabular}

\subsubsection{Processo de Execução da Pesquisa}

O processo de execução, em aderência aos objetivos específicos, encontra-se representado na Figura 1. 


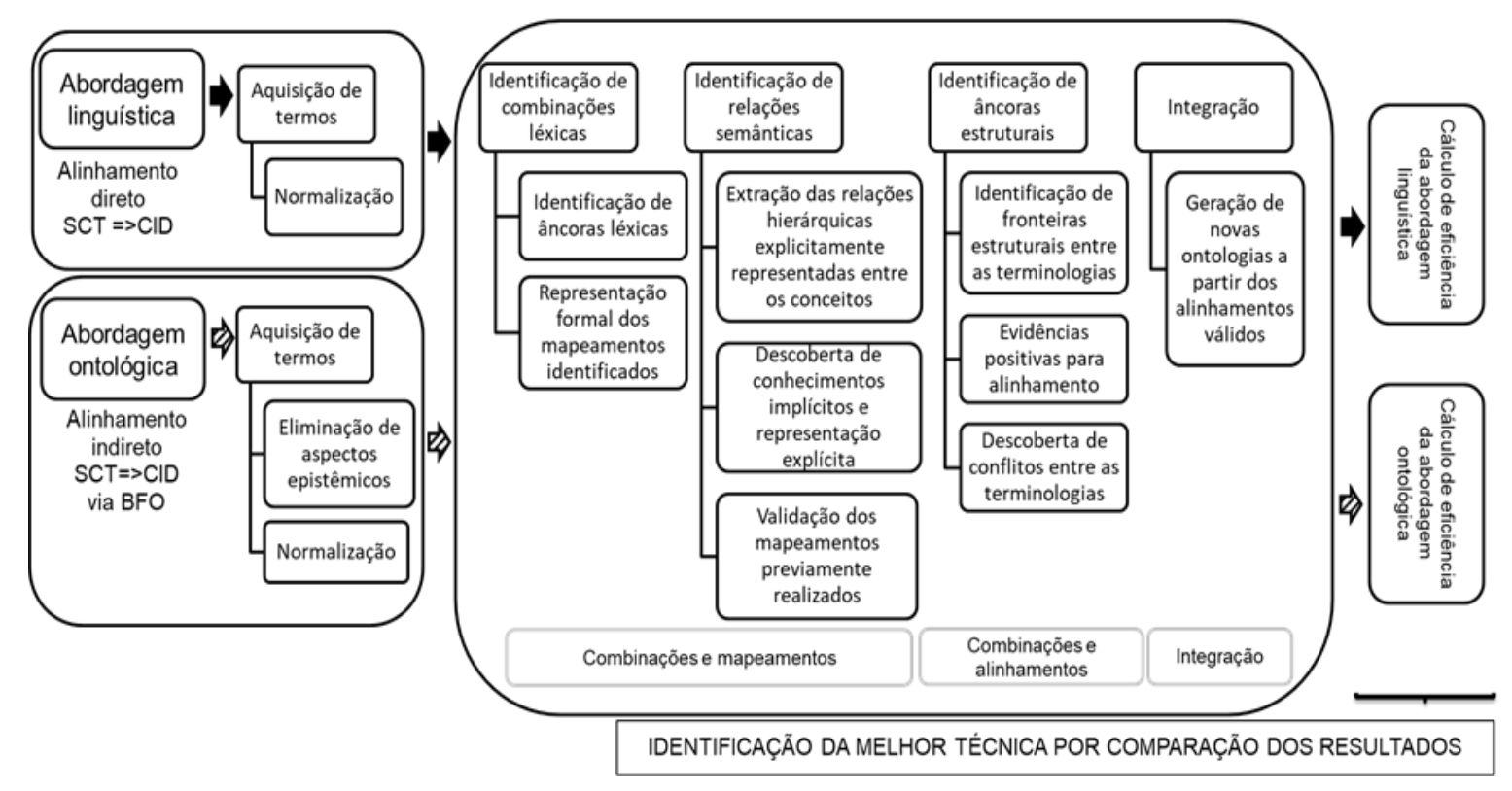

Figura 1. Processo de execução da pesquisa

A eficiência de cada abordagem para prover interoperabilidade semântica entre as terminologias clínicas será calculada como uma medida da proporção de evidências positivas para alinhamentos sobre o número total de combinações encontradas, conforme a equação:

$$
\begin{aligned}
\text { Eficiência }= & \text { (número de evidências positivas para alinhamento } \\
& / \text { total de combinações encontradas }) \times 100 .
\end{aligned}
$$

\section{CONCLUSÃO}

O diferencial da pesquisa está na oportunidade de explicar como elementos epistêmicos presentes nas terminologias clínicas conduzem à definição de classes que não atendem à princípios de classificação bem fundamentados, impactando negativamente na comparação de termos para fins de alinhamento, mapeamento, integração e evolução.

Além disso, espera-se demonstrar que o uso de princípios ontológicos, uma vez que são usados para orientar a construção de taxonomias livres de erros, possibilita um aumento nas chances de descobertas precisas de âncoras léxicas, de relações semânticas e âncoras estruturais que caracterizem fronteiras compartilhadas de conhecimento entre as terminologias clínicas. Abre-se assim espaço para a definição de alinhamentos com economia de tempo e de recursos de processamento, em função da realização de comparações apenas de termos ontologicamente identificados.

O artefato ontológico poderá ser utilizado de forma embarcada em sistemas de informação, visando prover interoperabilidade semântica sobre os dados dos pacientes, adquiridos durante a prestação de cuidados de saúde em suas diversas especialidades.

\section{AGRADECIMENTO}

A pesquisa é subsidiada pela Coordenação de Aperfeiçoamento de Pessoal de Nível Superior (CAPES). 


\section{REFERÊNCIAS}

ABNT, 2016.Informática em saúde: princípios para mapeamento entre sistemas terminológicos. Norma ABNT ISO/TR 12300:2016: Norma ABNT ISO/TR. [s.1.] Associação Brasileira de Normas Técnicas - ABNT.

Bodenreider, O., 2018. Evaluating the Quality and Interoperability of Biomedical Terminologies. [s.1.] LHNCBC Board of Scientific Counselors.

Bodenreider, O. et al., 2004. The Ontology-Epistemology Divide: A Case Study in Medical Terminology. Formal ontology in information systems: Proceedings of the ... International Conference. FOIS (Conference), v. 2004,p. 185.

Euzenat, J.; Shvaiko, P., 2007.Ontology Matching. Berlin, Heidelberg: Springer-Verlag, 2007.

Jansen, L., 2008. Chapter 7: Classifications. In: Applied Ontology: An Introduction [Hardcover]. 8. ed. German: Katherine Munn e Barry Smith, 2008. v. 9p. 342.

Ochieng, P.; Kyanda, S., 2018. Large-Scale Ontology Matching. ACM Computing Surveys, v. 51, n. 4, p. 1-35.

Rodrigues, J-M. et al., 2015.Semantic Alignment between ICD-11 and SNOMED CT. Studies in Health Technology and Informatics, v. 216, p. 790-794.

Schulz, S. et al., 2007. SNOMED CT's problem list: Ontologists' and logicians' therapy suggestions. Studies in health technology and informatics, v. 129, p. 802-806.

2017. Interface Terminologies, Reference Terminologies and Aggregation Terminologies: A Strategy for Better Integration. Studies in health technology and informatics, v. 245, p. 940-944.

, 2019. Standards in Healthcare Data. In: Kubben, P.; Dumontier, M.; Dekker, A. (Eds.). Fundamentals of Clinical Data Science. Cham: Springer International Publishing, 2019. p. 19-36.

Smith, B., 2003. Ontology. In: The Blackwell Guide to the Philosophy of Computing and Information. Oxford: Luciano Floridi, 2003.

2008. Ontology (Science). Formal Ontology in Information Systems: Proceedings of the Fifth International Conference (FOIS 2008). Anais: 170. In: Formal Ontology in Information Systems Frontiers in Artificial Intelligence And Applications. IOS Press, 2008.

Smith, B. et al., 2007. The OBO Foundry: coordinated evolution of ontologies to support biomedical data integration. Nature biotechnology, v. 25, n. 11, p. 1251

Zhang, S.; Bodenreider, O., 2005. Alignment of multiple ontologies of anatomy: deriving indirect mappings from direct mappings to a reference. AMIA ... Annual Symposium proceedings. AMIA Symposium, p. 864-868. 